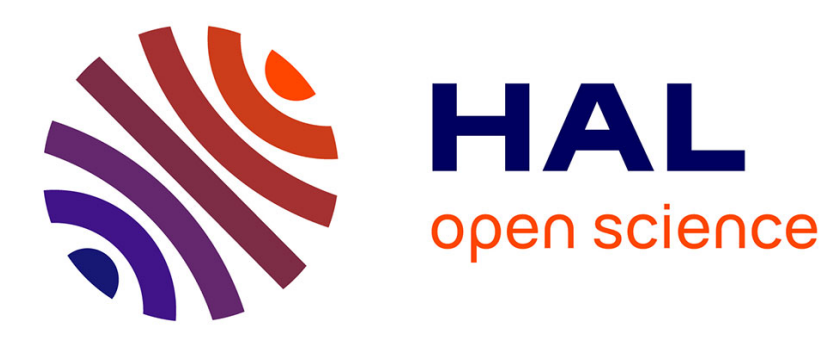

\title{
Core--Shell Nanocuboid Dimers with Nanometric Gaps
}

Markus Krug, Israa Haidar, Iman Ragheb, Joachim R Krenn, Andreas Hohenau, Viktor Kapetanovic, Matthieu Bugnet, Guillaume Radtke, Gianluigi A Botton, Georges Lévi, et al.

\section{- To cite this version:}

Markus Krug, Israa Haidar, Iman Ragheb, Joachim R Krenn, Andreas Hohenau, et al.. CoreShell Nanocuboid Dimers with Nanometric Gaps. Journal of Physical Chemistry C, 2020, 124 (34), pp.18690-18697. 10.1021/acs.jpcc.0c03830 . hal-03036822

\section{HAL Id: hal-03036822 \\ https://hal.science/hal-03036822}

Submitted on 2 Dec 2020

HAL is a multi-disciplinary open access archive for the deposit and dissemination of scientific research documents, whether they are published or not. The documents may come from teaching and research institutions in France or abroad, or from public or private research centers.
L'archive ouverte pluridisciplinaire HAL, est destinée au dépôt et à la diffusion de documents scientifiques de niveau recherche, publiés ou non, émanant des établissements d'enseignement et de recherche français ou étrangers, des laboratoires publics ou privés. 
archives-ouvertes

\section{Core-Shell Nanocuboid Dimers with Nanometric Gaps}

Markus Krug, Israa Haidar, Iman Ragheb, Joachim Krenn, Andreas Hohenau, Viktor Kapetanovic, Matthieu Bugnet, Guillaume Radtke, Gianluigi Botton, Georges Lévi, et al.

\section{To cite this version:}

Markus Krug, Israa Haidar, Iman Ragheb, Joachim Krenn, Andreas Hohenau, et al.. Core-Shell Nanocuboid Dimers with Nanometric Gaps. Journal of Physical Chemistry C, American Chemical Society, 2020, 124 (34), pp.18690-18697. 10.1021/acs.jpcc.0c03830 . hal-03036822

\section{HAL Id: hal-03036822 \\ https://hal.archives-ouvertes.fr/hal-03036822}

Submitted on 2 Dec 2020

HAL is a multi-disciplinary open access archive for the deposit and dissemination of scientific research documents, whether they are published or not. The documents may come from teaching and research institutions in France or abroad, or from public or private research centers.
L'archive ouverte pluridisciplinaire HAL, est destinée au dépôt et à la diffusion de documents scientifiques de niveau recherche, publiés ou non, émanant des établissements d'enseignement et de recherche français ou étrangers, des laboratoires publics ou privés. 


\title{
Core-Shell Nanocuboid Dimers with Nanometric Gaps
}

\author{
Markus Krug, Israa Haidar, Iman Ragheb, Joachim R. Krenn, Andreas Hohenau, Viktor Kapetanovic, \\ Matthieu Bugnet, Guillaume Radtke, Gianluigi A. Botton, Georges Lévi, Leila Boubekeur-Lecaque,* \\ and Nordin Felidj*
}

Cite This: https://dx.doi.org/10.1021/acs.jpcc.0c03830

Read Online

ACCESS | Lلll Metrics \& More | 回 Article Recommendations | st Supporting Information

ABSTRACT: Nanometric gaps in plasmonic structures can lead to huge optical near fields and, related, to strongly enhanced interaction of molecules and light. Nanocavities formed by sphere-on-film or cube-on-film systems were recently established as promising systems, eventually reaching the strong coupling regime. However, such structures are limited with respect to being bound to a surface and by having no means of adjusting the resonance wavelength to the requirements of arbitrary analytes, independent of the gap width. We suggest and investigate in this paper silver-gold compound nanocuboid dimers in colloidal solution as potential

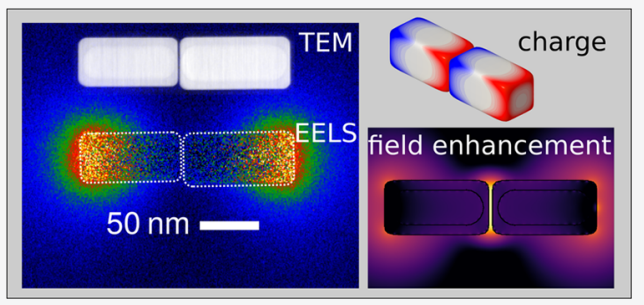
structures to mitigate both limitations. We analyze the dimers' plasmonic properties by a combination of optical spectroscopy, electron energy loss spectroscopy, and numerical simulations, focusing on the longitudinal dimer geometry and the dominant light-coupled plasmon mode. We then calculate optical field enhancements under light and electron excitation to assess the cuboid dimer's potential in sensing and spectroscopy applications.

\section{INTRODUCTION}

Plasmonic structures that contain nanometer gaps support modes with very large optical near-field enhancements localized in the gap. They thus constitute potentially attractive platforms for pushing the limits of surface-enhanced effects, including surface-enhanced Raman scattering or surfaceenhanced fluorescence. Applications thereof range from chemical identification of single molecules ${ }^{1}$ to directional emission of single photon emitters ${ }^{2}$ with applications in quantum computing. ${ }^{\frac{3}{3}}$ In recent years, the optical near-field enhancement in gaps formed by colloidal metal nanoparticles deposited on flat metal films, with some nanometric spacer in between, has attracted some attention. Well-known techniques for fabricating controlled planar spacer layers can be applied to realize minimal gap sizes, eventually reaching the subnanometer regime, which allowed us to demonstrate quantumplasmonic effects including optical field-driven electron tunneling, ${ }^{4}$ huge spontaneous emission enhancement, ${ }^{5}$ or strong coupling to single molecules at room temperature. ${ }^{6}$ However, particularly for ultrasensitive spectroscopic applications, often the analyte is highly diluted in a liquid and its detection probability is then limited by diffusion. ${ }^{7}$ It is thus beneficial to distribute the plasmonic gap structures in the solvent volume instead of being surface-bound as in the particle-on-film systems.

Colloidal nanocubes (silver, gold, or core-shell geometries thereof) can be dimerized to form thickness-controlled, atomically flat gaps, similar to particle-on-film systems. Due to their flat faces, just a few discrete mutual orientations are possible, improving the control of dimerization, in contrast to, e.g., nanorods that can dimerize with a continuous range of binding angles. ${ }^{8}$ The plasmonic coupling of nanocubes has been thoroughly investigated,, 10 in particular for their longitudinal bonding modes and for their transverse cavity plasmons (TCPs) in the gap. ${ }^{11,12}$ Nanocubes in close proximity lead to further peculiar effects, such as Fano-induced transparency ${ }^{13-15}$ and charge transfer plasmons. ${ }^{16}$ However, nanocube dimers offer only limited spectral tunability that is dominated by a strong gap width dependence of the plasmonic resonances responsible for the large optical near-field enhancements. To overcome this limitation, we use in this work cuboid-shaped instead of cube-shaped Au-core Ag-shell nanoparticles, as reported for the monomer case in ref 17 . Nanocuboid dimers, to the best of our knowledge not studied before, show similar plasmonic modes to cube dimers, but with dipolar bonding modes that red-shift only little with decreasing gap distance, and, importantly, that can be tuned with the nanocuboid length as an additional design parameter.

In the following, by a combination of optical attenuance spectroscopy, electron energy loss spectroscopy (EELS) in a scanning transmission electron microscope (STEM), and numerical simulations, we first review the optical properties of nanocuboid monomers as a basis for understanding the properties of nanocuboid dimers. We then focus on the

Received: May 1, 2020

Revised: August 4, 2020

Published: August 4, 2020 
longitudinal dimer geometry to calculate and analyze the optical near-field enhancement of the dominant light-coupled gap mode, as well as electron-beam-induced optical near fields. Finally, we discuss the cuboid dimer's potential in sensing and spectroscopic applications.

\section{EXPERIMENTAL AND THEORETICAL METHODS}

Chemicals. Hydrogen tetrachloroaurate trihydrate $\left(\mathrm{HAuCl}_{4}, 3 \mathrm{H}_{2} \mathrm{O}\right.$ 99.9\%) was purchased from Alfa Aesar. Hexadecyltrimethylammonium bromide (CTAB, 98\%), hexadecyltrimethylammonium chloride (CTAC, >95\%), and sodium oleate $(\mathrm{NaOL},>97 \%)$ were purchased from TCI Chemicals. Silver nitrate $\left(\mathrm{AgNO}_{3},>99 \%\right)$, L-ascorbic acid (AA, $>99 \%$ ), and sodium borohydride (99\%) were purchased from Sigma-Aldrich. All solutions were prepared with Milli-Q water with resistivity $>18.2 \mathrm{M} \Omega \mathrm{cm}^{-1}$.

Synthesis of Gold Nanorods (AuNRs). AuNRs were synthesized using seed-mediated growth methods reported previously. ${ }^{18}$ Briefly, the seed solution was prepared by a fast reduction of $\mathrm{HAuCl}_{4}(0.5 \mathrm{mM})$ in $10 \mathrm{~mL}$ of $\mathrm{CTAB}$ solution $(0.2 \mathrm{M})$ with $\mathrm{NaBH}_{4}(0.6 \mathrm{~mL}$ of fresh $0.01 \mathrm{M}$ solution $)$ under vigorous stirring. The solution color turned from yellow to brownish. After $2 \mathrm{~min}$, the seed solution was left undisturbed at room temperature for $30 \mathrm{~min}$ before addition in the growth medium. A growth solution containing CTAB $(3.5 \mathrm{~g}, 0.037 \mathrm{M})$ and $\mathrm{NaOL}(0.617 \mathrm{~g})$ in $125 \mathrm{~mL}$ of water was thermostated at $30{ }^{\circ} \mathrm{C}$ before the addition of $6 \mathrm{~mL}$ of $\mathrm{AgNO}_{3}(4 \mathrm{mM})$ solution. After $15 \mathrm{~min}, 125 \mathrm{~mL}$ of a $1 \mathrm{mM} \mathrm{HAuCl}_{4}$ solution was added under stirring at $700 \mathrm{rpm}$. When the solution became colorless (after about $90 \mathrm{~min}$ ), $1.05 \mathrm{~mL}$ of $\mathrm{HCl}$ (37 wt \% in water) was injected. After $15 \mathrm{~min}$ of stirring at $400 \mathrm{rpm}$, ascorbic acid (1.25 mL of $0.064 \mathrm{M}$ solution) was added and vigorously stirred $(1000 \mathrm{rpm})$ for $30 \mathrm{~s}$ before the addition of $400 \mu \mathrm{L}$ of a $\mathrm{Au}$ seed solution. This resulting growth solution was stirred for $30 \mathrm{~s}$ and left undisturbed at $30{ }^{\circ} \mathrm{C}$ overnight. AuNRs were purified by centrifugation at $6000 \mathrm{rpm}$ for $30 \mathrm{~min}$ and then at $5800 \mathrm{rpm}$ for $10 \mathrm{~min}$. CTAB (6 mL, $1 \mathrm{mM}$ solution) was then added before undergoing another centrifugation at $5800 \mathrm{rpm}$ for $10 \mathrm{~min}$.

Synthesis of Core-Shell Au@Ag Cuboids. The asprepared gold-CTAB nanorods ( $2 \mathrm{~mL}, 0.39 \mathrm{nM}$ solution) were diluted in $23 \mathrm{~mL}$ of CTAC solution $(19 \mathrm{mM})$ and heated to 60 ${ }^{\circ} \mathrm{C}$. After $20 \mathrm{~min}, \mathrm{AgNO}_{3}(2 \mathrm{mM}, 6 \mathrm{~mL})$ and $6 \mathrm{~mL}$ of a mixture solution containing AA $(50 \mathrm{mM})$ and CTAC (35 $\mathrm{mM}$ ) were simultaneously injected by a dual-syringe pump at a flow rate of $1 \mathrm{~mL} / \mathrm{min}$. After $2 \mathrm{~h}$ of stirring, the final product was collected by centrifugation at $5800 \mathrm{rpm}$ for $10 \mathrm{~min}$ and washed with the CTAC solution $(1 \mathrm{mM})$ twice, and finally redispersed in water. The nanocuboids are terminated by six stable $\{100\}$ facets with final outer dimensions of $84 \pm 7 \mathrm{~nm}$ along the long axis and $44 \pm 4 \mathrm{~nm}$ along the short axis and an aspect ratio of about 1.9, as depicted in Figure 1a-c. For size distribution, see Figure $S 1$ in the Supporting Information.

Assembly of Au@Ag Cuboids in Solution. $\mathrm{Au} @ \mathrm{Ag}$ cuboids $(100 \mu \mathrm{L})$ were suspended in $200 \mu \mathrm{L}$ of acetonitrile. TPyLA ligand ( $1 \mu \mathrm{L}$ of $1 \mathrm{mM}$ solution in acetonitrile; for chemical structure, see Figure S2 in the Supporting Information) was then added to the $\mathrm{Au} @ \mathrm{Ag}$ cuboid suspension. The assembly in solution was triggered by the addition of cadmium ions ( $1 \mu \mathrm{L}$ of $4 \mathrm{mM}$ solution) forming a chelate complex and monitored by UV-vis attenuance and Raman spectroscopy.

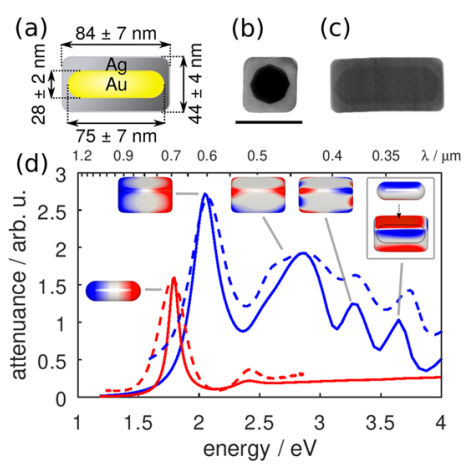

Figure 1. (a) Sketch of a Au@Ag nanocuboid. Bright-field STEM images of an (b) upright and (c) reclined nanocuboid. The scale bar indicates $50 \mathrm{~nm}$. (d) Measured (dashed blue line) and simulated (solid blue line) attenuance of an aqueous solution of $\mathrm{Au} @ \mathrm{Ag}$ cuboids. For comparison, the solid and dashed red lines depict the measured and simulated attenuance spectra, respectively, of an aqueous solution of the Au nanorods used as seed. The insets show the surface charge distributions calculated at the peak positions of the simulated spectra for the nanocuboid plasmonic modes (dipolar longitudinal resonance at $2.05 \mathrm{eV}$ or $605 \mathrm{~nm}$, transverse resonance at $2.88 \mathrm{eV}$ or $430 \mathrm{~nm}$, multipolar resonance at $3.26 \mathrm{eV}$ or $380 \mathrm{~nm}$, transverse resonance with mirror charges on the gold core at $3.65 \mathrm{eV}$ or $340 \mathrm{~nm})$ and the longitudinal dipolar nanorod mode $(1.78 \mathrm{eV}$ or $695 \mathrm{~nm}$ ). For comparison, the simulated (solid red) and measured (dashed red line) attenuance spectra of an aqueous solution of $\mathrm{Au}$ nanorods used as seed particles (core) are plotted.

Optical Attenuance Spectroscopy. Optical attenuance spectra were recorded from the samples in a quartz cuvette (path length, $2 \mathrm{~mm}$ ) with a bright-field optical microscope (Nikon, TI-DH) equipped with a spectrometer (Andor, SR303i) and a CCD camera (ANDOR, DU401A-BR-PD) through a $100 \times$ objective (Nikon, NA 0.7) using a halogen lamp as the light source. UV-vis-NIR absorption spectra were recorded with an Agilent 8453 spectrophotometer in the range of $190-1100 \mathrm{~nm}$, using $2 \mathrm{~mm}$ path length quartz cuvettes.

Electron Energy Loss Spectroscopy (EELS). EELS experiments have been carried out in STEM mode in a double-corrected FEI Titan Cubed 80-300 (S)TEM operated at $80 \mathrm{kV}$ equipped with a monochromator and a GIF Quantum ERS imaging filter. The monochromator was adjusted to reach an energy resolution of $90 \mathrm{meV}$. The spectra were acquired with a dispersion of $0.01 \mathrm{eV} /$ channel. In STEM-EELS, an electron beam is raster scanned across the sample and electron energy loss spectra are recorded for each beam position. Subsequently, three-dimensional data cubes $(x, y, E)$ are obtained, from which two-dimensional maps for specific loss energies are sliced. Post-acquisition deconvolution with the Richardson-Lucy algorithm was applied using the SpectrumImagingAnalysisPy Python program. This improved the effective energy resolution by $10 \mathrm{meV}$ after one iteration. ${ }^{19,20}$ EELS spectra were deconvoluted with a reference spectrum obtained by averaging over an empty substrate area. The energy and contrast ranges were user-defined with SpectrumImagingAnalysisPy to optimally isolate particular plasmon modes in the EELS maps. For the EELS on nanocuboid dimers, the dimers are formed randomly by capillary action during the deposition of a drop of monomer solution on the 15 $\mathrm{nm}$ thick $\mathrm{Si}_{3} \mathrm{~N}_{4}$ membrane of a TEM grid. The interparticle distance (gap) is $3 \mathrm{~nm}$, as determined from STEM-HAADF micrographs. 
Numerical Simulations. Simulations were done with the MNPBEM toolbox, ${ }^{21}$ a solver for Maxwell's equations based on a boundary element method. ${ }^{22}$ Nanoparticle boundaries are thereby defined as polygon meshes. Dielectric functions for gold and silver were taken from Johnson and Christy, ${ }^{23} n=$ 1.33 and 1.34 were used for water and acetonitrile, respectively. For the monomers, the CTAC coating was neglected. For the dimers with the enhanced near-field concentration in the gap, the CTAC coating was accounted for using an effective refractive index of the surrounding of $1.38^{24}$ instead of 1.33 . The discretization, i.e, the size of the mesh faces, was chosen so that the simulations converge, with a finer mesh in the gap region of the dimers. For the simulation of STEM-EELS results, both nanocuboid monomers and dimers were assumed to be in a homogeneous environment with $\epsilon_{\mathrm{m}}=2.1$ to take into account the substrate-induced red shift caused by placing the nanocuboids on a $15 \mathrm{~nm}$ thick $\mathrm{Si}_{3} \mathrm{~N}_{4}$ membrane $\left(\epsilon_{\mathrm{Si}_{3} \mathrm{~N}_{4}}=\right.$ 4.1). A detailed discussion of the effect of the substrate, the acceleration voltage, and the gold core on EELS can be found in Supporting Information Figures S6-S10. EELS maps were constructed by evaluating the EEL probability for every electron beam position on a grid for a fixed loss energy. The electron energy was set to $80 \mathrm{keV}$ as in the experiment.

\section{RESULTS AND DISCUSSION}

We start with the characterization of the cuboid-shaped coreshell Au@Ag nanoparticles (nanocuboids) that are later used as building blocks for the dimerization. The nanocuboids used in this work were obtained by seed-mediated growth (see the Experimental and Theoretical Methods section) of silver on gold nanorods of $75 \pm 7 \mathrm{~nm}$ length and $28 \pm 2 \mathrm{~nm}$ diameter. They are terminated by six stable $\{100\}$ facets with final outer dimensions of $84 \pm 7 \mathrm{~nm}$ along the long axis and $44 \pm 4 \mathrm{~nm}$ along the short axis, as depicted in Figure 1a-c. Dispersed in water, they show multiple strong plasmonic resonances throughout the visible spectrum (Figure 1d, dashed blue line), in stark contrast to the optical attenuance spectrum of the gold nanorods (Figure 1d, dashed red line) used as core. The latter is dominated by the longitudinal dipolar resonance at $1.78 \mathrm{eV}(695 \mathrm{~nm})$, and the transverse dipolar resonance is visible only as a weak spectral peak at $2.41 \mathrm{eV}(514 \mathrm{~nm})$. To assign particular plasmon modes to the peaks in the nanocuboid spectrum, we first simulate the optical attenuance cross section with the MNPBEM toolbox (see the Experimental and Theoretical Methods section). To account for the random cuboid orientation in solution, we apply a directionand polarization-averaged illumination in the simulations. Generally, the simulations (Figure 1d, solid red and blue lines) are in excellent agreement with the experimental spectra. The larger width of the experimental plasmon peaks compared to the simulation is most likely due to a small but intrinsic size distribution in the cuboid solution.

The simulated charge maps calculated for excitation energies corresponding to the attenuance peaks allow us to identify their mode character (insets in Figure 1d). We find that the dominant attenuance peak at $2.05 \mathrm{eV}(605 \mathrm{~nm})$ stems from the longitudinal dipolar resonance. The transverse dipolar resonance appears at $2.88 \mathrm{eV}(430 \mathrm{~nm})$, and the peak at $3.26 \mathrm{eV}(380 \mathrm{~nm})$ is due to a multipolar edge mode of first order, asymmetrically coupled between left and right end faces of the cuboid. Finally, the resonance at $3.65 \mathrm{eV}(340 \mathrm{~nm})$ is specific for the $\mathrm{Ag} @ \mathrm{Au}$ material combination, reflecting transversal mirror charges on the gold nanorod core that oscillate out of phase with the nanocuboid surface charges. These mirror charges appear in the spectral overlap region of the inter- and intraband regions of $\mathrm{Au}$ and $\mathrm{Ag}$, respectively, ${ }^{17,25}$ where $\operatorname{Re}\left(\varepsilon_{\mathrm{Ag}}\right)<0$, but $\operatorname{Re}\left(\varepsilon_{\mathrm{Au}}\right)>0$.

We now turn to the nanocuboid dimers. Experimentally, nanocuboid dimers are synthesized in solution by moleculemediated assembly of nanocuboid monomers (see the Experimental and Theoretical Methods section). In addition to the end-to-end geometry (Figure $2 \mathrm{a}, \mathrm{I}$ ) and in contrast to

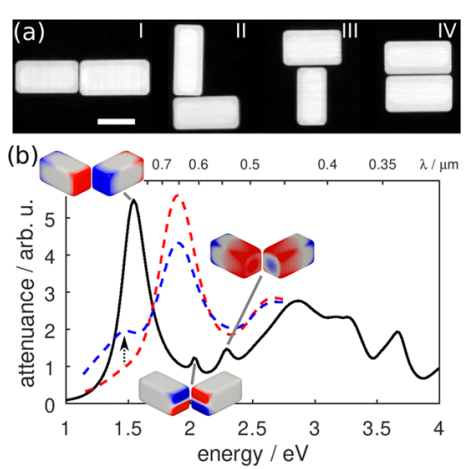

Figure 2. (a) STEM images of different configurations of nanocuboid dimers. (b) Optical attenuance spectra of the colloidal solution of nanocuboids in acetonitrile before (dashed red line) and after the addition of the molecular linker (dashed blue line), showing a diminished dipolar peak and the emergence of a dimer peak at 1.47 $\mathrm{eV}(845 \mathrm{~nm})$ indicated by the arrow. This peak corresponds to the simulated bonding mode of an end-to-end nanocuboid dimer for a gap separation of $3 \mathrm{~nm}$ (solid black line). The insets depict surface charge distributions of the nanocuboid dimer (rotated to reveal the surface charges in the gap) calculated at the indicated peak positions. The simulated attenuance spectra of other dimer configurations can be found in Supporting Information Figure S3.

gold nanorod dimers, ${ }^{8,26}$ only three further dimer configurations are found, leading to a mixture of end-to-end, side-byside, and T- and L-shaped configurations (Figure 2a,I-IV). This is due to the rather homogeneous distribution of the CTAC surfactant on the cuboid surface, enabling to link the cuboids just for discrete orientations with parallel faces, thus maximizing van der Waals interactions between alkyl chains. Centrifugation $^{27}$ and electrophoresis ${ }^{28}$ processes could be applied to obtain monodisperse solutions of a particular dimer configuration.

The nanocuboid dimerization is accompanied by a significant modification of the optical response, as shown in the attenuance spectra in Figure $2 \mathrm{~b}$. Compared to the spectrum of a single nanocuboid colloidal solution (dashed red line), the attenuance spectrum after initiating the dimerization (dashed blue line) shows a reduced dipolar peak at $1.91 \mathrm{eV}(650 \mathrm{~nm})$ and another LSPR mode emerging at $1.47 \mathrm{eV}(845 \mathrm{~nm})$. When two Au@Ag cuboids are brought within a close distance upon dimerization, their optical response is modified by electromagnetic interaction. In a first, rough model, the dipolar LSPRs of the two nanocuboids hybridize in phase or out of phase, i.e., bonding or antibonding, to form new dimer modes with a lower or higher energy than the uncoupled modes on individual nanocuboids. ${ }^{29}$ However, this simplified view is only valid for relatively large distances that ensure dominant dipolar coupling. Particularly at low gap widths of only a few nanometers as in our case, strong coupling 
of LSPRs on both particles leads to complex hybridization between multiple dipolar and multipolar modes. ${ }^{29,30}$ As detailed below, we thus find hybrid modes with large field concentration in the gap that cannot be identified with just one particular mode of the individual particles.

By comparing the experimental and simulated spectra in Figure $2 \mathrm{~b}$, we can attribute the experimentally observed attenuance peak at $1.47 \mathrm{eV}$ that emerges upon dimerization partly to the longitudinal dipole bonding LSPR (corresponding to in-phase dipole oscillations of the individual cuboids) of the end-to-end dimer (1.53 eV in the simulations). Within the gap region, the surface charge density at this resonance (inset) is rather homogeneous, in contrast to the charge densities at the end faces of a single cuboid (inset in Figure 6). This points toward the contribution of multipolar modes, as discussed in the previous paragraph, but can also be described as a coupling of the bonding dipole mode with the lowest order $((1,1)$, the numbers denote the horizontal and vertical extrema of the surface charge distributions) transverse cavity plasmon (TCP) mode of the gap region, ${ }^{31}$ in a simplistic view. Further peaks in the simulated spectrum can be attributed to a dipolar $((2,1)$ and $(1,2))$ TCP $(2.02 \mathrm{eV})$ and the antibonding longitudinal dipole mode $(2.28 \mathrm{eV})$ with some out-of phase contribution of a $(1,1)$ TCP mode (see charge distributions in the insets of panel c). Both peaks can be excited due to retardation effects at inclined incidence, but are not observed in the experiment as the residual nanocuboid monomer dipolar peak dominates in this spectral range.

To summarize so far, we synthesized Au@Ag nanocuboid dimers in colloidal solution and attributed the main features observed in the attenuance spectra to specific modes of the end-to-end configuration. The largest attenuance peak is caused by a bonding dipole mode, whose calculated charge distribution shows a signature of coupling to the lowest order TCP with a remarkably homogeneous charge density throughout the gap region.

In view of potential sensor applications, we now analyze the optical near-field enhancement achievable by optical excitation with the help of simulations. We first review the $|E|^{2}$ enhancements achievable with the nanocuboid monomers. The random orientation of the nanocuboids in aqueous solution with respect to a certain illumination direction leads to an averaging in the experimental spectra. In the simulations, we can take this into account by averaging over many different incidence angles and polarizations with respect to the nanocuboid orientation. Generally, we find that directional averaging leads to near-field enhancement values that are on the same order of magnitude as the enhancement value for the most efficient far-field coupling direction. We thus plot $|E|^{2} / \mid$ $\left.E_{0}\right|^{2}$ only for the particular incidence direction (perpendicular to the long axis) and polarization (parallel to the long axis) that optimally excites the longitudinal dipolar mode. In Figure 3a, the optical near-field enhancements are compared to the attenuance cross section. The LSPR resonance positions of the far-field spectrum (light blue line) are found to coincide with those of the respective near-field spectrum (dark blue line), but with a slight red shift of the latter. This effect is a universal property of a damped harmonic oscillator and increases with the damping of the system. ${ }^{32}$

Selected near-field maps of the first two modes are plotted in Figure $3 \mathrm{~b}-\mathrm{d}$. The $y-z$ plane maps $1.5 \mathrm{~nm}$ from the end facets in panels c (longitudinal dipole) and $\mathrm{d}$ (transversal dipole) are somewhat similar from a simple inspection, as the highest field
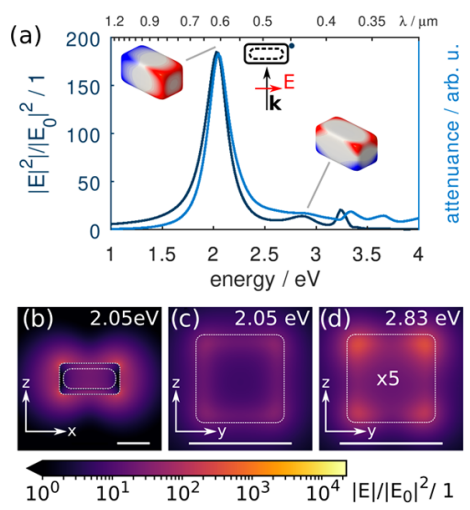

Figure 3. Simulated near-field intensity enhancement $|E|^{2} /\left|E_{0}\right|^{2}$ upon light excitation for a nanocuboid monomer. (a) Spectral dependence of intensity enhancement (dark blue line) and normalized attenuance (light blue line), for a position close to the nanocuboid corner, $1.5 \mathrm{~nm}$ from the surface (blue dot, inset), where the electric fields are maximum (compare (c) and (d)). Near-field maps for (b) the longitudinal dipolar mode at $2.05 \mathrm{eV}(605 \mathrm{~nm})$ in the $x z$-plane through the particle center and (c) in the $y z$-plane, $1.5 \mathrm{~nm}$ away from the end face. (d) Near-field pattern of the transverse dipolar mode at $2.88 \mathrm{eV}(430 \mathrm{~nm})$ in the $y z$-plane. The spectra and electric field maps are done for illumination in the $z$-direction, with polarization in the $x$ direction. Note the logarithmic color scale. The scale bars indicate 50 $\mathrm{nm}$. The insets depict surface charge distributions of the marked modes.

enhancement occurs close to the corners in both cases. However, a closer analysis reveals that the near fields of the longitudinal dipolar mode point mainly in the $x$-direction (i.e., out of the drawing plane), whereas those of the transversal dipolar mode also have a substantial $z$-component, as to be expected from the surface charge distribution (insets in Figure 3a). The largest local intensity enhancements are found to be in the range of $|E|^{2} /\left|E_{0}\right|^{2} \simeq 200$.

For the nanocuboid dimer, the results of the optical nearfield calculations are depicted in Figure 4. For the bonding

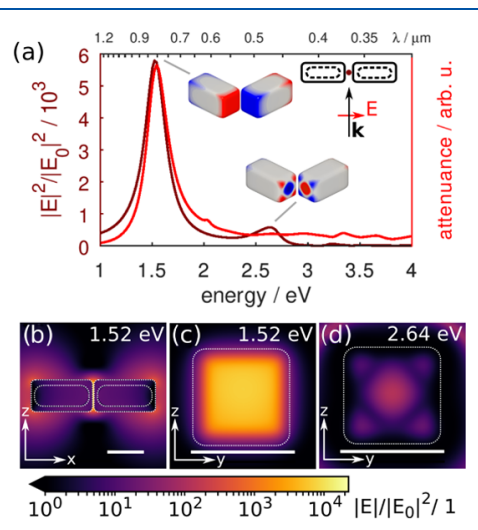

Figure 4. Simulated near-field enhancement $|E|^{2} /\left|E_{0}\right|^{2}$ upon light excitation for a nanocuboid dimer. (a) Spectral dependence of intensity enhancement (dark red line) and normalized attenuance (light red line), for the midgap position with $3 \mathrm{~nm}$ gap separation (red dot, inset). Near-field maps for (b) the bonding mode $(1.52 \mathrm{eV})$ in the $x y$-plane through the particle's center and (c) in the $y z$-plane in midgap. (d) Near-field map of the TCP mode at $2.64 \mathrm{eV}(466 \mathrm{~nm})$ in the $y z$-plane in midgap. The spectra and electric field maps are done for illumination in the $z$-direction, with polarization in the $x$-direction. Note the logarithmic color scale. The scale bars indicate $50 \mathrm{~nm}$. The insets depict surface charge distributions of the marked modes. 
dipolar mode excited at $1.52 \mathrm{eV}$, the electric field distribution at the end faces (Figure $4 \mathrm{~b}$ ) is nearly identical to that of the monomer close to its outer face (compare Figure $3 \mathrm{~b}$ ). It is within the $3 \mathrm{~nm}$ wide gap that the field enhancement is boosted by a factor of more than 30 beyond the monomer case. The intensity enhancement of the dimer bonding mode reaches a factor of nearly 6000 (5500 for directional averaging), and the $|E|^{2} /\left|E_{0}\right|^{2}$ maps show that the enhancement is very homogeneous throughout the gap region, which is in contrast to the very localized near-field enhancements observed in nanorod dimers ${ }^{8}$ or bow-tie configurations, ${ }^{33}$ and may be advantageous for sensing applications.

The second strongest peak in the near-field enhancement spectrum at $2.64 \mathrm{eV}$ (Figure 4a) stems from a superposition of the $(3,1)$ and $(1,3)$ TCP modes excited at equal strength (this mode is similar to the " $\mathrm{B}$ "-mode for nanocube dimers in ref 31 ). In the present case, the intensity enhancement is about 500 , i.e., 1 order of magnitude lower than that for the bonding dipole mode.

To proceed from these results closer to the main aim of this work, i.e., to find a solution-based system with tunable gap resonances with high field enhancement and little gap width dependence, we depict in Figure 5 the dependence of the

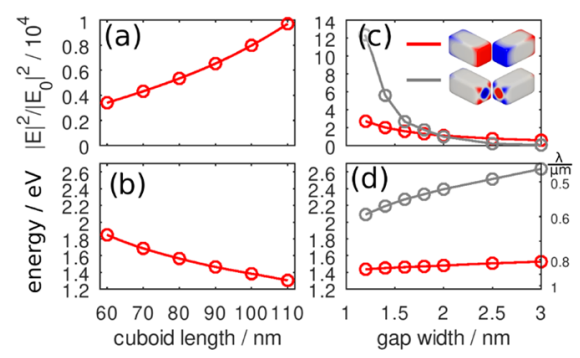

Figure 5. Dependence of the near-field intensity enhancement in the gap and the resonance position of the bonding dipole mode on $(a, b)$ the cuboid length and (c, d) the gap width (solid red lines). For comparison, the gap width dependence for the $(3,1)+(1,3)$ TCP mode is plotted in (c, d) with the gray lines. The insets show the corresponding surface charge maps.

bonding dipole resonance on gap width and the cuboid length. When changing the length of the cuboids, for example, from 60 to $120 \mathrm{~nm}$ (Figure 5b), the resonance shifts from 1.8 to $1.2 \mathrm{eV}$, demonstrating the desired tunability. At the same time, the near-field intensity enhancement maintains the same magnitude, increasing only by a factor of 3 (Figure 5a). When changing the gap width, we find only weak changes in resonance position and near-field intensity enhancement. This is in stark contrast to the TCP modes. For example, the TCP $(3,1)+(1,3)$ mode shows a 172 -fold increase of its near-field intensity enhancement (Figure 5c) and a $0.55 \mathrm{eV}$ resonance energy shift (Figure 5d) when the gap width is reduced from 3 to $1.2 \mathrm{~nm}$.

To complement the optical and near-field characterization, STEM-EELS experiments were performed on individual nanocuboid monomers and dimers deposited on the $\mathrm{Si}_{3} \mathrm{~N}_{4}$ membrane. In EELS, a fast electron passing the nanoparticle acts as a local but broad-band source, exciting the plasmonic resonances. Due to the accompanying energy loss of the electron, distinct peaks are observed in the electron energy loss spectra $^{34}$ and can in this respect be compared to the measured optical spectroscopic and simulated near-field data.
For the monomer (Figure 6), we find a reasonable agreement between experiment and simulation, particularly
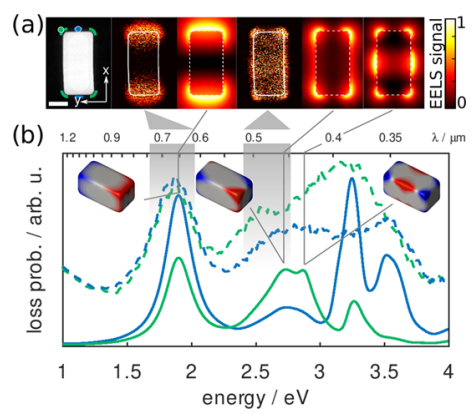

Figure 6. EELS measurements and simulations for a single Au@Ag nanocuboid. (a) From left to right: HAADF-STEM image, the colored dots mark where the spectra plotted in (b) were acquired; experimental EELS map integrated from 1.71 to $2.01 \mathrm{eV}$; simulated EELS map at $1.85 \mathrm{eV}$; experimental EELS map integrated from 2.41 to $2.78 \mathrm{eV}$; simulated EELS maps at 2.60 and $2.95 \mathrm{eV}$. The color maps are adjusted to the maximum electron loss probability in each panel, and the scale bar indicates $25 \mathrm{~nm}$. (b) Experimental EEL spectra (dashed lines) integrated over the regions around the corners (green) or along the edges (blue) as indicated in (a), and simulated EEL spectra (solid lines) for the electron beam positions as indicated in (a) by the blue and green dots. The inset shows the surface charge maps of the longitudinal $(1.90 \mathrm{eV})$ and transversal dipolar plasmon modes (2.73 eV, asymmetrically coupled between left and right cuboid faces). Peaks at higher energies can be attributed to multipolar modes at $2.90,3.24$, and $3.50 \mathrm{eV}$ and the mirror charge mode at about $3.60 \mathrm{eV}$ (compare Figure 1). The longitudinal dipolar resonance is well reproduced by the simulations; however, above $3 \mathrm{eV}$, the accordance between simulations and experiment is limited. We attribute this to the effects caused by the substrate and the surfactant layer. See the Supporting Information for EELS maps of an upright cuboids end face (Figure S4) and more information on the effect of the substrate (Figure S7).

in the spectral region below $2.5 \mathrm{eV}$. The dominant peak there is associated with the longitudinal dipolar mode at $1.9 \mathrm{eV}$, which is corroborated by the measured and simulated EELS maps (Figure $6 \mathrm{~b}, \mathrm{c}$ ). Further peaks can be attributed to the transversal dipole resonance $(2.73 \mathrm{eV})$ multipolar resonances (2.90, 3.24, and $3.50 \mathrm{eV}$; the surface charge plots of the latter two resonances can be found in Supporting Information Figure S6) and a mirror charge mode $(3.60 \mathrm{eV})$. The LSPR peaks are shifted to lower energies compared to the optical spectra in water (Figure 1) due to the presence of the membrane substrate. For energies above $3 \mathrm{eV}$, the accordance between simulation and experiment is rather limited, which we assign to the influence of the substrate ${ }^{35}$ and the surfactant layer, not taken into account in the simulations.

For the cuboid dimer, the results are depicted in Figure 7. The measured EELS maps of the bonding and antibonding dimer modes at energy losses of 1.37 and $2.08 \mathrm{eV}$ (Figure $7 \mathrm{a}, \mathrm{b})$ compare well to the simulated maps (Figure $7 \mathrm{c}, \mathrm{d}$ ). Both dimer modes show distinct patterns in the EELS maps, which can be readily understood through their mode symmetry. The bonding mode (panels a and c) features charges of opposite sign across the gap (inset, panel c) that cannot be excited by the electron beam in the center of the gap. This is expected since the excitation is mirror symmetric with respect to the gap center but the electric fields of the bonding dipole mode are antisymmetric. The bonding dipolar mode has strong optical near fields in the gap; however, they are dominantly $x$ - 


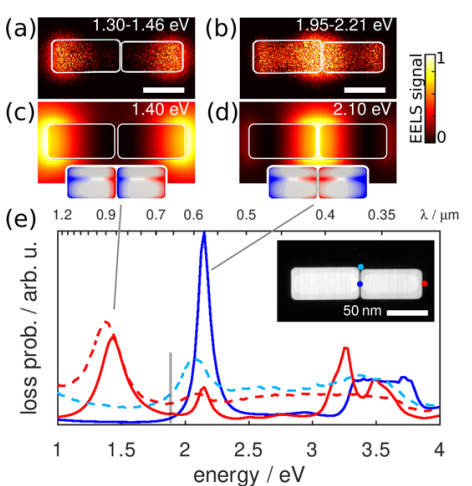

Figure 7. EELS measurements and simulations for a $\mathrm{Ag} @ \mathrm{Au}$ nanocuboid dimer with a gap separation of $3 \mathrm{~nm}$. (a, b) Experimental and $(c, d)$ simulated EELS maps. The insets show the corresponding surface charge maps of the bonding and antibonding plasmon modes. The color maps are adjusted to the maximum electron energy loss probability in each panel. The scale bar indicates $50 \mathrm{~nm}$. (e) Experimental (dashed lines) and simulated (solid lines) EEL spectra for the electron beam positions indicated by the colored dots in the HAADF-STEM image in the inset. The gray vertical line at $1.9 \mathrm{eV}$ indicates the dipolar resonance position of the monomer to highlight the coupling-induced mode splitting of the bonding and antibonding dipolar mode. In contrast to the simulation, the experimental spectra were measured outside the gap for a better signal-to-noise ratio, which is responsible for the lower intensity of the antibonding mode peak. The simulations are done for a homogeneous dielectric medium $\left(\epsilon_{\mathrm{m}}=\right.$ 2.1 ), whereas in the experiment, the particles are in vacuum and supported by a $15 \mathrm{~nm}$ thick $\mathrm{Si}_{3} \mathrm{~N}_{4}$ membrane $\left(\epsilon_{\mathrm{Si}_{3} \mathrm{~N}_{4}}=4.1\right)$. Further measured and simulated EELS maps of nanocuboid dimers can be found in Supporting Information Figure S5.

polarized (i.e., along the dimer axis) and have only little impact on the energy loss of the perpendicularly propagating electrons in EELS. ${ }^{34,36}$ Recent developments in tomographic 3D imaging could be applied to overcome this limitation. ${ }^{35,37}$ On the other hand, the antibonding mode (panels $b$ and $d$ ) is very well excited by an electron beam passing through the gap; however, this mode has identical charge densities on the opposite faces of the gap and thus only weak optical near fields within the gap. A comparison of the EELS spectra recorded at selected positions (Figure 7) with respect to their resonance positions with the monomer dipolar resonance position of 1.90 $\mathrm{eV}$ (gray line) further illustrates the coupling-induced mode splitting.

The interaction between LSPRs and analyte molecules within regions of high LDOS can be studied using STEMEELS. For example, one could think about a situation where the optical absorption properties of molecules within a nanometric volume shall be probed by STEM-EELS; however, the direct EELS signal of the molecules is too low and their direct exposure to the swift electron beam would cause damage to them. If the molecules were positioned, instead, in the gap of our nanocuboid dimer, the electron beam could be placed at an outer facet of one nanocuboid, thereby efficiently exciting the bonding dipolar mode and exposing the analyte molecule to a large electromagnetic field. Such a configuration could translate the molecule's optical properties to the EELS spectrum, which in turn could be used to characterize the molecules and simultaneously avoid beam damage.

In the following, we analyze the nanocuboid dimer along these lines. The used surfactant and linker molecules, however, show no pronounced feature in their optical spectra within the relevant spectral range; thus, we cannot expect to observe clear features of LSPR-mediated coupling between the electron beam and optical excitations of the linker molecules. Changing the linker molecules would go beyond the scope of this work, so we restrict ourselves to simulations where we calculate the electron beam-induced electric field intensity as a measure of the interaction strength and compare relative numbers at selected positions (Figure 8). The near-field maps are

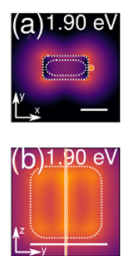

(c)
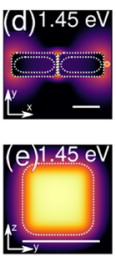

$(\mathrm{f})$

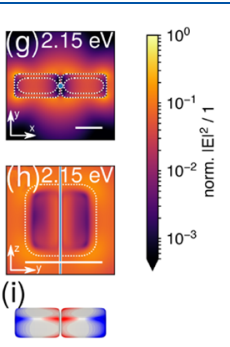

Figure 8. (a) Simulated normalized near-field intensity upon electron beam excitation. The electron beam is placed at the optimal position for the excitation of a given mode (compare Figures 6 and 7). Longitudinal dipolar mode $(1.90 \mathrm{eV})(\mathrm{a})$ in the $x-y$ plane at half particle height and (b) in the $y-z$ plane, $1.5 \mathrm{~nm}$ away from the end face. Longitudinal dipole bonding mode $(1.45 \mathrm{eV})(\mathrm{d})$ in the $x-y$ plane at half particle height and (e) in the $y-z$ plane in midgap. Longitudinal dipole antibonding mode $(2.15 \mathrm{eV})(\mathrm{g})$ in the $x-y$ plane at half particle height and (h) in the $y-z$ plane in midgap. (c, $\mathrm{f}, \mathrm{i})$ The corresponding surface charge maps. Note the logarithmic color scale. The scale bars indicate $50 \mathrm{~nm}$.

simulated for a single position of the electron beam (orange or blue dots and lines in Figure $8 \mathrm{a}-\mathrm{h}$ ), which is close to its optimum position, as identified from the EELS maps (Figures 6 and 7). Indeed, for the excitation of the dipolar mode of the single cuboid, the map of the electron beam-induced near fields (Figure 8a,b) qualitatively resembles the near-field enhancement for optical excitation (Figure $3 b, c$ ) when the immediate region around the electron beam is excluded. However, for an excitation with a plane wave, the field enhancement far from the nanoparticle approaches unity. In contrast, as the electric field of an electron beam decreases with $1 / r^{2}$, the electric field intensity increases toward zero far from the particle.

With this in mind, we consider the electron beam close to the end faces of dimers (orange dot, Figure 8d) due to the ease at which the bonding mode is excited there (compare Figure $7 c)$. In agreement with the familiar bonding mode near-field pattern (Figure $4 b, c)$, the electric fields are well confined to the gap and rather weak at the outside. Conversely, for the antibonding mode, the electron beam-induced near-field intensities (Figure 8g,h) in the gap are rather small. Nevertheless, the comparably large near-field intensities induced by the electron beam in the case of the dimer's bonding dipole mode could render it useful to carry out EELS on molecules positioned in the gap. Moreover, the optical near fields in the gap are rather homogeneous within the gap region and the spectral position of the corresponding bonding dipolar LSPR can be designed by changing the nanocuboid length to a desired position.

\section{CONCLUSIONS}

In view of their potential as building blocks in enhanced optical or electron beam spectroscopy, we investigated the far- and near-field properties of colloidal Au@Ag cuboid monomers 
and dimers by optical spectroscopy, EELS, and numerical simulations. Evidencing distinct plasmon modes such as multipolar resonances and mirror charges in the gold nanorod core for the single $\mathrm{Au} @ \mathrm{Ag}$ cuboid, special attention was paid to the dimer case and its dominantly excitable bonding and antibonding dipole modes. We find that the bonding dipole mode is accompanied by strong optical near-field enhancements in the gap region with a homogeneous profile along the gap. A similar situation can be observed for the case of electron-beam-induced optical near fields. The large optical near-field intensities in combination with their homogeneity throughout the gap and their spectral tunability suggest that such systems have a high potential when applied for the realization of surface-enhanced optical and EELS sensors, including phenomena where strong coupling between light and molecules is involved. However, the challenge of getting arbitrary analytes into a distance-controlled gap remains to be solved.

\section{ASSOCIATED CONTENT}

\section{SI Supporting Information}

The Supporting Information is available free of charge at https://pubs.acs.org/doi/10.1021/acs.jpcc.0c03830.

Chemical structure of the linker molecule, additional spectra and EELS maps of all different cuboid dimer configurations, and an analysis of the effects of the core and the substrate on EELS and electron-beam-induced near fields (PDF)

\section{AUTHOR INFORMATION}

\section{Corresponding Authors}

Leila Boubekeur-Lecaque - Université de Paris, ITODYS, CNRS, F-75006 Paris, France; 이이이.org/0000-0001-5832310X; Email: leila.boubekeur@univ-paris-diderot.fr.

Nordin Felidj - Université de Paris, ITODYS, CNRS, F-75006

Paris, France; (1) orcid.org/0000-0002-3243-2671;

Email: nordin.felidj@univ-paris-diderot.fr.

\section{Authors}

Markus Krug - Institute of Physics, University of Graz, 8010 Graz, Austria; orcid.org/0000-0002-3563-1721

Israa Haidar - Universite de Paris, ITODYS, CNRS, F-75006 Paris, France

Iman Ragheb - Université de Paris, ITODYS, CNRS, F-75006 Paris, France

Joachim R. Krenn - Institute of Physics, University of Graz, 8010 Graz, Austria

Andreas Hohenau - Institute of Physics, University of Graz, 8010 Graz, Austria; (1) orcid.org/0000-0002-9034-3044

Viktor Kapetanovic - Department of Materials Science and Engineering and the Canadian Centre For Electron Microscopy, McMaster University, Hamilton, Ontario L8S4M1, Canada

Matthieu Bugnet - University of Lyon, INSA-Lyon, UCBL Lyon 1, MATEIS, UMR 5510 CNRS, 69621 Villeurbanne, France; Department of Materials Science and Engineering and the Canadian Centre For Electron Microscopy, McMaster University, Hamilton, Ontario L8S4M1, Canada; (1) orcid.org/0000-0002-8272-8964

Guillaume Radtke - Sorbonne Université, Muséum National d'Histoire Naturelle, UMR CNRS, 75005 Paris, France; Institut de Minéralogie, de Physique des Materiaux et de Cosmochimie, 75005 Paris, France
Gianluigi A. Botton - Department of Materials Science and Engineering and the Canadian Centre For Electron Microscopy, McMaster University, Hamilton, Ontario L8S4M1, Canada; Canadian Light Source, Saskatoon, SK S7N 2V3, Canada; (i) orcid.org/0000-0002-8746-1146

Georges Lévi - Université de Paris, ITODYS, CNRS, F-75006 Paris, France

Complete contact information is available at:

https://pubs.acs.org/10.1021/acs.jpcc.0c03830

\section{Notes}

The authors declare no competing financial interest.

\section{ACKNOWLEDGMENTS}

This work was supported by the CNRS, the University ParisDiderot and Sorbonne Paris Cite. ANR (Agence Nationale de la Recherche) and CGI (Commissariat à l'Investissement d'Avenir) are gratefully acknowledged for their financial support of this work through Labex SEAM (Science and Engineering for Advanced Materials and devices) ANR-11LABX-086, and the funded project ANR-11-IDEX-05-02. Part of this work was supported by Austrian Science Fund FWF Project P25034. The STEM-EELS work was performed at the Canadian Centre for Electron Microscopy, a national facility supported by the Canada Foundation for Innovation through the MSI program, NSERC, and McMaster University. The authors thank Ulrich Hohenester and Andreas Trügler for helpful discussions and support with the MNPBEM simulations.

\section{REFERENCES}

(1) Le Ru, E. C.; Etchegoin, P. G. Single-Molecule SurfaceEnhanced Raman Spectroscopy. Annu. Rev. Phys. Chem. 2012, 63, 65-87.

(2) Wang, D.; Zhu, W.; Best, M. D.; Camden, J. P.; Crozier, K. B. Directional Raman Scattering from Single Molecules in the Feed Gaps of Optical Antennas. Nano Lett. 2013, 13, 2194-2198.

(3) Aharonovich, I.; Englund, D.; Toth, M. Solid-state Single-Photon Emitters. Nat. Photonics 2016, 10, 631.

(4) Zhu, W.; Esteban, R.; Borisov, A. G.; Baumberg, J. J.; Nordlander, P.; Lezec, H. J.; Aizpurua, J.; Crozier, K. B. Quantum Mechanical Effects in Plasmonic Structures with Subnanometre Gaps. Nat. Commun. 2016, No. 11495.

(5) Hoang, T.; Akselrod, G. M.; Mikkelsen, M. H. Ultrafast RoomTemperature Single Photon Emission from Quantum Dots Coupled to Plasmonic Nanocavities. Nano Lett. 2016, 270-275.

(6) Chikkaraddy, R.; de Nijs, B.; Benz, F.; Barrow, S. J.; Scherman, O. A.; Rosta, E.; Demetriadou, A.; Fox, P.; Hess, O.; Baumberg, J. J. Single-Molecule Strong Coupling at Room Temperature in Plasmonic Nanocavities. Nature 2016, 127-130.

(7) Sheehan, P. E.; Whitman, L. J. Detection Limits for Nanoscale Biosensors. Nano Lett. 2005, 5, 803-807.

(8) Shao, L.; Woo, K. C.; Chen, H.; Jin, Z.; Wang, J.; Lin, H.-Q. Angle- and Energy-Resolved Plasmon Coupling in Gold Nanorod Dimers. ACS Nano 2010, 4, 3053-3062.

(9) Goris, B.; Guzzinati, G.; Fernández-López, C.; Pérez-Juste, J.; Liz-Marzán, L. M.; Trügler, A.; Hohenester, U.; Verbeeck, J.; Bals, S.; Tendeloo, G. V. Plasmon Mapping in Au@Ag Nanocube Assemblies. J. Phys. Chem. C 2014, 118, 15356-15362.

(10) Bordley, J. A.; Hooshmand, N.; El-Sayed, M. A. The Coupling between Gold or Silver Nanocubes in Their Homo-Dimers: A New Coupling Mechanism at Short Separation Distances. Nano Lett. 2015, $15,3391-3397$. 
(11) Knebl, D.; Hórl, A.; Trügler, A.; Kern, J.; Krenn, J. R.; Puschnig, P.; Hohenester, U. Gap plasmonics of silver nanocube dimers. Phys. Rev. B 2016, 93, No. 081405.

(12) Esteban, R.; Aguirregabiria, G.; Borisov, A. G.; Wang, Y. M.; Nordlander, P.; Bryant, G. W.; Aizpurua, J. The Morphology of Narrow Gaps Modifies the Plasmonic Response. ACS Photonics 2015, 2, 295-305.

(13) Grillet, N.; Manchon, D.; Bertorelle, F.; Bonnet, C.; Broyer, M.; Cottancin, E.; Lerme, J.; Hillenkamp, M.; Pellarin, M. Plasmon Coupling in Silver Nanocube Dimers: Resonance Splitting Induced by Edge Rounding. ACS Nano 2011, 5, 9450-9462.

(14) Pellarin, M.; Ramade, J.; Rye, J. M.; Bonnet, C.; Broyer, M.; Lebeault, M.-A.; Lerme, J.; Marguet, S.; Navarro, J. R. G.; Cottancin, E. Fano Transparency in Rounded Nanocube Dimers Induced by Gap Plasmon Coupling. ACS Nano 2016, 10, 11266-11279.

(15) Yang, Z.; Wang, M.; Song, X.; Deng, J.; Yao, X. Engineering the plasmonic optical properties of cubic silver nanostructures based on Fano resonance. J. Chem. Phys. 2013, 139, No. 164713.

(16) Tan, S. F.; Wu, L.; Yang, J. K.; Bai, P.; Bosman, M.; Nijhuis, C. A. Quantum Plasmon Resonances Controlled by Molecular Tunnel Junctions. Science 2014, 343, 1496-1499.

(17) Tebbe, M.; Kuttner, C.; Mayer, M.; Maennel, M.; Pazos-Perez, N.; König, T. A.; Fery, A. Silver-Overgrowth-Induced Changes in Intrinsic Optical Properties of Gold Nanorods: From Noninvasive Monitoring of Growth Kinetics to Tailoring Internal Mirror Charges. J. Phys. Chem. C 2015, 119, 9513-9523.

(18) Ye, X.; Zheng, C.; Chen, J.; Gao, Y.; Murray, C. B. Using Binary Surfactant Mixtures To Simultaneously Improve the Dimensional Tunability and Monodispersity in the Seeded Growth of Gold Nanorods. Nano Lett. 2013, 13, 765-771.

(19) Bellido, E.; Rossouw, D.; Botton, G. Toward $10 \mathrm{meV}$ Electron Energy-Loss Spectroscopy Resolution for Plasmonics. Microscopy and Microanalysis 2014, 20, 767-778.

(20) icbicket, icbicket/DataAnalysis: SpectrumImagingAnalysisPy v1.0.0, 2017. https://doi.org/10.5281/zenodo.807763.

(21) Hohenester, U.; Trügler, A. MNPBEM - A Matlab Toolbox for the Simulation of Plasmonic Nanoparticles. Comput. Phys. Commun. 2012, 183, 370

(22) de Abajo, F. G.; Howie, A. Retarded Field Calculation of Electron Energy Loss in Inhomogeneous Dielectrics. Phys. Rev. B 2002, 65, No. 115418.

(23) Johnson, P. B.; Christy, R. W. Optical Constants of the Noble Metals. Phys. Rev. B 1972, 6, 4370-4379.

(24) Lin, L.; Zhonghui, L.; Xiyao, L.; Gu, H.; Ye, J. Nanoscale 2017, 9, 2213.

(25) Zhang, S.; Bao, K.; Halas, N. J.; Xu, H.; Nordlander, P. Substrate-Induced Fano Resonances of a Plasmonic Nanocube: A Route to Increased-Sensitivity Localized Surface Plasmon Resonance Sensors Revealed. Nano Lett. 2011, 11, 1657-1663.

(26) Haidar, I.; I; L'evi, G.; Aubard, L. M. J.; Lau-Truong, S.; Grand, J.; Neuville, D.; F'elidj, N.; Boubekeur-Lecaque, L. Highly Stable Silica-Coated Gold Nanorods Dimers for Solution-Based SERS. Phys. Chem. Chem. Phys. 2016, 18, 32272-32280.

(27) Tyler, T. P.; Henry, A.-I.; Duyne, R. P. V.; Hersam, M. C. Improved Monodispersity of Plasmonic Nanoantennas via Centrifugal Processing. J. Phys. Chem. Lett. 2011, 2, 218-222.

(28) Hanauer, M.; Pierrat, S.; Zins, I.; Lotz, A.; Sönnichsen, C. Separation of Nanoparticles by Gel Electrophoresis According to Size and Shape. Nano Lett. 2007, 7, 2881-2885.

(29) Nordlander, P.; Oubre, C.; Prodan, E.; Li, K.; Stockman, M. I. Plasmon Hybridization in Nanoparticle Dimers. Nano Lett. 2004, 4, 899-903.

(30) Kim, M.; Kwon, H.; Lee, S.; Yoon, S. Effect of Nanogap Morphology on Plasmon Coupling. ACS Nano 2019, 13, 1210012108 .

(31) Knebl, D.; Hörl, A.; Trügler, A.; Kern, J.; Krenn, J. R.; Puschnig, P.; Hohenester, U. Gap plasmonics of silver nanocube dimers. Phys. Rev. B 2016, No. 081405.
(32) Zuloaga, J.; Nordlander, P. On the Energy Shift between NearField and Far-Field Peak Intensities in Localized Plasmon Systems. Nano Lett. 2011, 11, 1280-1283.

(33) Sundaramurthy, A.; Crozier, K. B.; Kino, G. S.; Fromm, D. P.; Schuck, P. J.; Moerner, W. E. Field Enhancement and Gap-Dependent Resonance in a System of Two Opposing Tip-to-Tip Au Nanotriangles. Phys. Rev. B 2005, 72, No. 165409.

(34) Hohenester, U.; Ditlbacher, H.; Krenn, J. R. Electron-EnergyLoss Spectra of Plasmonic Nanoparticles. Phys. Rev. Lett. 2009, 103, No. 106801.

(35) Nicoletti, O.; de la Peña, F.; Leary, R. K.; Holland, D. J.; Ducati, C.; Midgley, P. A. Three-Dimensional Imaging of Localized Surface Plasmon Resonances of Metal Nanoparticles. Nature 2013, $502,80-84$.

(36) Losquin, A.; Kociak, M. Link between Cathodoluminescence and Electron Energy Loss Spectroscopy and the Radiative and Full Electromagnetic Local Density of States. ACS Photonics 2015, 2, $1619-1627$

(37) Haberfehlner, G.; Schmidt, F.-P.; Schaffernak, G.; Hörl, A.; Trügler, A.; Hohenau, A.; Hofer, F.; Krenn, J. R.; Hohenester, U.; Kothleitner, G. 3D Imaging of Gap Plasmons in Vertically Coupled Nanoparticles by EELS Tomography. Nano Lett. 2017, 17, 67736777. 